\title{
A WIRE-CHAMBER SPECTROMETER FOR MUON SPIN ROTATION ${ }^{*}$ )
}

\author{
R. Tedeschi and P. Podini \\ CERN, Geneva, Switzerland \\ and \\ Università di Parma, Italy, and \\ Istituto Nazionale di Fisica Nucleare, Sez. Milano, Italy \\ R. De Renzi \\ Rutherford Appleton Laboratory, \\ Chilton, Oxfordshire, United Kingdom \\ A.P. Jeavons and R. Magnanini \\ CERN, Geneva, Switzerland \\ L.0. Nor1in \\ Institute of Physics, University of Uppsala, Sweden
}

\begin{abstract}
We report on the development of a spectrometer for Muon Spin Rotation based on multiwire proportional chambers. The coordinates provided by telescopes each composed of two chambers are used to reconstruct the muon and the positron trajectories in $\mu^{+} \rightarrow \mathrm{e}^{+}+v_{\mathrm{e}}+\bar{v}_{\mu}$ decay. A real-time analysis program verifies that the two tracks intersect within the target: this constitutes a very stringent test to veto events which do not correspond to a muon decay. The results of some experiments are given in order to illustrate the field of application of this spectrometer.
\end{abstract}

(Submitted to Nuclear Instruments and Methods in Physics Research)

*) Work supported by the Experimental Physics Division, CERN, Geneva, and by the Istituto Nazionale di Fisica Nucleare, Gruppo V, Milano. 


\section{INTRODUCTION}

Muon Spin Rotation ( $\mu \mathrm{SR}$ ) is nowadays a well-established experimental technique in solid-state physics and in chemistry. Its domain of investigation has expanded to almost all of the subjects typically covered by magnetic resonance techniques, where new information can be acquired using the muon as an interstitial magnetic probe. Furthermore $\mu S R$ can be exploited in all those cases in which the muon can be regarded as a light isotope of hydrogen: this aspect has given rise to radical reaction studies in organic compounds and to diffusion studies in crystals. The essence of the method, on whose standard techniques a review was recently published in this journal by Lankford et al. [1], is very simple. A spin polarized beam of muons is stopped in a sample. Owing to parity violation in the decay $\mu^{+} \rightarrow e^{+}+\nu_{e}+\bar{v}_{\mu}$, the direction of the positron moment carries information on the orientation of the muon spin. Thus the precession of the 1atter in a magnetic field perpendicular to the initial polarization can be monitored by recording the positron rate in a chosen direction, as a function of the time which has elapsed from the birth of the corresponding muon. Thus a $\mu$ SR experiment consists basically of measuring the lifetimes of the stopped muons. It is because of this intrinsic simplicity that, despite the still rising interest in $\mu S R$ applications, many aspects of its data-acquisition technique have remained almost invaried since the beginning. In particular, particle detection has always been carried out with telescopes of plastic scintillators, whose spatial resolution is 1 imited to the definition of the solid angle through which the sample is observed. This method of detection is certainly the most economical but it entails some intrinsic drawbacks. In this paper we will describe a new type of spectrometer developed at CERN that combines all the standard characteristics of conventional apparatuses with a good spatial resolution. This spectrometer was designed for continuous muon beams, such as the one available at the CERN $600 \mathrm{MeV}$ Synchro-cyclotron. The use of pulsed beams, even if compatible in principle with the detection system, would require major modifications to the logic of the data acquisition. 


\section{DRAWBACKS OF CONVENTIONAL EXPERIMENTS}

The time differential method of $\mu S R$ produces histograms where the number of events detected in a telescope is plotted against the interval between the incoming of a muon (START time) and its decay (STOP time).

The modulation frequency of the USR signal due to the spin precession ranges between $10^{4}$ and $10^{10} \mathrm{~Hz}$; therefore a relevant parameter for assessing the performance of a spectrometer is its time resolution. From this point of view scintillation detection is very good: a conventional system can easily have a 1 ns resolution, which gives a frequency cut-off at $500 \mathrm{MHz}$, and higher resolutions can be achieved with some effort. However, the poor spatial discrimination of scintillator counters impairs their ability to distinguish between signal and noise, where the latter is those events that triggex the spectrometer and are not muon decays in the sample. We shall describe the possible sources of this noise, making reference to its effects on the time histogram, whose analytic form is:

$$
N(t)=N_{0} \exp \left(-t / \tau_{\mu}\right)\left[1+\alpha P(t) \cos \left(\omega_{\mu} t+\phi\right)\right]+B
$$

Here $\tau_{\mu}$ is the average lifetime of the muon, $\alpha$ is an asymetry parameter, $P(t)$ is the time-dependent muon spin polarization, $\omega_{\mu}$ the angular velocity, $\phi$ the initial phase of the precession, and the term $B$ accounts for additional events not correlated with the muons (fig. 1). We can thus divide the noise into three main categories:

i) Spurious signals

The intrinsic momentum spread of the beam, the beam straggling introduced by the degrader, and the presence in the sample region of scintillators, cryostat walls and other parts of the apparatus imply that a significant number of particles are stopped outside the sample itself. The positrons coming from these muons contribute to the modulation of the time histogram with unwanted events that will sum to the term $P(t)$ of expression (1). 


\section{ii) Background}

Muon beams can be far from perfect as far as $\mu S R$ applications are concerned: they can be heavily contaminated by other particles, mainly pions and positrons. All these particles can be detected by the telescopes and are therefore a potential source of noise. There are different ways of avoiding their effects on the histogram: they can either be physically diverted by means of expensive devices (e.g. d.c. separators) or simply discriminated on the basis of their different momentum loss through the degrader material used to slow down muons. The last method, though, cannot eliminate all the contamination, mainly because the fast electronics needed to produce the vetoes can cope only with a limited pulse rate.

The only criterion for accepting or rejecting an event, once it has triggered the spectrometer, is that the time which has elapsed between the START pulse and the following STOP pulse should be within the range of the collected histogram. Therefore there will always be a fraction of the accepted events which do not correspond to muon decays; they will contribute to term $B$ in expression (1). If they originate from randomly distributed pulses they will show an exponential decay in time, whose decay parameter (equal to the random pulse rate) will generally be very long on the muon lifetime scale. If, however, their origin is correlated with a detected muon they can give to $B$ an appreciable time dependence. Although, in principle, the background can be exactly determined by fitting procedures and subsequently subtracted from the histogram, it enhances the statistical noise. The effect is larger in the last channels of the histogram, where the exponential decay has reduced the number of counts per channel to a level comparable to that of a typical background (a few per cent of $\mathrm{N}_{0}$ ). Therefore this problem will be particularly severe when measuring the decay parameter of a slow relaxation of the muon polarization.

\section{iii) Distortions}

The presence of pions in the beam can give additional noise problems. If these particles are stopped in the sample they will decay with an average lifetime of 26 ns. The resulting muons will have both randomly oriented spins and an 
indeterminacy in the time of birth of the order of the pion lifetime. Their HSR signal will distort the exponential form of expression (1) and it will reduce the asymmetry parameter $\alpha$. The criterion used to minimize this effect is usually based on counting rate and asymmetry measurements to optimize the degrader thickness. However, with scintillator spectrometers this method is indirect and time consuming.

The three types of noise described above can be drastically reduced by introducing a detection system capable of checking the spatial correlation of an event. For this purpose we added a pair of wire chambers to the START and STOP telescopes; the coordinates provided by them allow the determination of the muon and positron trajectories. The intersection of these tracks, or vertex, is the place where the muon decayed. The spurious signals mentioned in point (i) can now be eliminated by the requirement that the vertex of an accepted event must be within the sample volume. The background described in (ii) will be greatly reduced, because pulses, which are random in their time distribution, will have an equally random spatial distribution. Furthermore the ability to represent the three-dimensional density of vertices in the space provided a quick "photographic" way of optimizing the degrader thickness, which is a considerable improvement on the standard solution outlined in (iii).

\section{THE SPECTROMETER}

\subsection{The wire chambers}

One START and two STOP telescopes are provided. A schematic layout of a telescope of the spectrometer is given in fig. 2a. The reader can compare its main features with the corresponding scheme for a conventional apparatus which is given in $\mathrm{fig} .2 \mathrm{~b}$.

Wire-chamber construction follows traditional techniques [2]. Each chamber consists of two cathode planes of $70 \mu \mathrm{m}$ diameter $\mathrm{Be} / \mathrm{Cu}$ wires, and one anode plane of $10 \mu \mathrm{m}$ diameter gold-plated tungsten wires. Anode and cathode wire spacing is $1 \mathrm{~mm}$ and the interplane gap is $3 \mathrm{~mm}$. The gas mixture is composed of $70 \%$ argon-freon (with freon in $1 \%$ proportion) and $30 \%$ isobutane; $40 \%$ of the argon-freon is bubbled 
through a bottle of isopropyl alcohol. The chambers are operated with the anode planes at $+3.0 \mathrm{kV}$ and the cathode planes earthed.

Two-dimensional read-out is obtained by detecting the cluster of positive pulses on the orthogonal cathode planes and calculating the centre [3]. The cathode wires are joined in pairs and each pair is connected to an amplifier and a discriminator. Priority encoders locate the ends $(\alpha, \beta)$ of the cluster, and a hard-wired processor checks the width $(\alpha-\beta)$ of the cluster for validity, and then calculates the centre $[(\alpha+\beta) / 2]$. This read-out method is simple and fast. The intrinsic resolution of $\pm 1 \mathrm{~mm}$ is adequate for this application. The number of channels may be reduced substantially by coding techniques, and the calculation eliminated by using look-up tables [4].

An event in a telescope is defined by a coincidence between the scintillators and the wire chambers. This causes the time-to-digital converter (TDC) to store a time value in its controller, and wire-chamber coordinates to be recorded in their respective chamber controllers.

\subsection{The time-to-digital converter}

In conventional $\mu S R$ spectrometers there is no way to check whether a given pair of START and STOP pulses belongs to the same muon decay: as we mentioned in the previous section the only restriction imposed is that the time that has elapsed between the two pulses should be less than a preset maximum value which is usually $10 \mu \mathrm{s}$. Under these conditions it is necessary to reject those events in which two muons are present in the sample at the same time. This is generally done by sending a veto signal to the TDC: once a START pulse is accepted, if a second START arrives before the STOP pulse, the clock is reset. As a consequence a TDC used in conventional experiments has to measure only one time interval at a time. When the wire chamber information is added, it becomes possible to distinguish, among several incoming muons, which one is correlated with a detected positron. Therefore the TDC must be able to handle multi-START events. The model that we designed operates on the following principles:

i) Every pulse is labelled according to the telescope of origin (START $=0, \operatorname{STOP}_{1}=1$, $\left.\operatorname{STOP}_{2}=2\right)$. 
ii) The TDC measures the elapsed time between two consecutive pulses, irrespective of their origin: each pulse causes the output of a free running counter to be recorded.

iii) An overflow flag is set if the time that has elapsed between two pulses is longer than $32 \mu s$; this flag is automatically cleared by the next pulse, unless, of course, the overflow condition occurs again.

The recorded number and its complement are fed into a set of adders to provide the time difference between the present and the previous pulses. About $150 \mathrm{~ns}$ after the arrival of each pulse the code and time information is passed, via the TDC controller, to three different sets of first-in-first-out buffers (FIFOs), depending on the origin of the pulse (START, STOP 1, STOP $_{2}$ ).

The counter is driven by a $125 \mathrm{MHz}$ quartz oscillator and a cable interpolator is used to obtain a 2 ns time resolution $[5,6]$. This finite time resolution determines a cut-off on the detectable frequencies, but it is not the only factor contributing to the passband of the spectrometer. Another contribution comes from the fact that the incoming START and STOP pulses are not synchronized with the oscillator period: this determines an uncertainty in the measured time interval, and the corresponding passband curve can be taken into account analytically [7]. The total passband of the apparatus, however, depends also on the time jitter that affects the pulses from the detectors. From this point of view wire chambers are not very good compared to fast scintillators and we decided to take the timing pulses that drive the TDC from one of the phototube detectors coupled to each telescope, gated by the corresponding set of coincidences, as is shown in fig. 2a. The phototube pulse is derived from a constant fraction discriminator so that the total time jitter introduced by the electronics (discriminator plus following coincidence unit) is negligible $(<2 \mathrm{~ns})$. The remaining jitter is due to the different transit times of light pulses coming from opposite ends of the scintillator: this can be estimated to be of the order of $0.5 \mathrm{~ns}--$ much smaller than the other effects. 


\subsection{Data transfer}

A block diagram of the data flow is given in fig. 3. Every event in each telescope creates a set of four spatial values -- the two pairs of wire chamber coordinates in byte form packed into two 16-bit words, plus a 16-bit word containing the time information. These data are presented by the TDC and chamber controller units to a common bus line. Whenever a set of data is ready, a flag is set, one for each telescope, which will activate the execution of a microprogram in the "data flow controllex" interface. This microprogram is selectable. Its standard version with the double positron-telescope spectrometer operates as follows:

1) If a START flag is set the data words are read into a circular buffer where the data of the $n$ latest START pulses are present, $n$ being preset by software.

2) If the STOP flag is set, the microcode compiles a FIFO buffer in the following order:

i) Four 16-bit words containing the STOP address, the time information, and the four coordinates.

ii) $3 n$ words containing the time and spatial information for the $\mathrm{n}$ latest START events coming from the circular buffer.

This set of $(4+3 n)$ words is transferred in direct memory access (DMA) mode into a PDP 11/04, where the final buffer is formed. Once the buffer is full -- it holds typically 500 sets -- it is sent via CAMAC to a PDP 11/60 for analysis. The 11/04 acts also as a compiler for the microprograms that govern the "data flow controller" interface.

\subsection{The vertex reconstruction}

The coordinates supplied by two pairs of wire chambers (START and STOP telescopes) together with the wire-chamber positions are sufficient information to define two straight lines in space, the $\mathrm{e}^{+}$and $\mu^{+}$tracks, which should define a vertex. In a real experiment, however, these tracks do not generally intersect owing to the finite spatial resolution of the spectrometer. The distance between 
the two reconstructed trajectories can be as large as ten times the basic wirechamber resolution $( \pm 1 \mathrm{~mm})$, depending on the geometry of the spectrometer and on the operating conditions. As a consequence, appropriate data analysis must estimate this distance, prior to providing the coordinates of a vertex.

The geometrical problem of evaluating the distance between two lines in space is straightforward but time consuming, since it involves the resolution of a $3 \times 3$ determinant [8]. An approximate but faster algorithm has been implemented, based on the principle illustrated in fig. 4. Given two straight lines $\mathrm{m}$ and $\mathrm{n}$, there are always two planes $\mu$ and $\beta$ containing them, both orthogonal to a chosen third plane $\gamma$. Moreover, the intersection of the $\mu$ and $\beta$ planes is a line $h$, of course i.tself orthogonal to the same plane $\gamma$. Denoting by A the intersection point of lines $m$ and $h$ and by $B$ the intersection point of the lines $n$ and $h$, we define a pseudo distance as the absolute value $d \equiv|A-B|$. An orthogonal right-handed reference frame can now be chosen so that the $\gamma$ plane is defined by the $x$ and $y$ axes and the $z$ axis is parallel to the line $h$. The resulting algorithm to evaluate $d$ and the coordinates of $A$ and $B$ is trivial and involves a few multiplications and divisions. The geometry of the experiment is such that the $\mu^{+}$tracks lie close to the $\mathrm{y}$ direction, while the positron telescopes are centred around the $\mathrm{x}$ direction; as a consequence the pseudo distance $d$ is a good approximation to the real distance, of which it is in fact the projection along the z-axis.

In the present configuration of the spectrometer, for geometrical reasons, the muon telescope allows a more accurate reconstruction of the particle trajectory than the positron telescopes. For this reason the vertex is chosen to be the intersection of the $\mu^{+}$track with the direction of the pseudo distance (point A in fig. 4).

\subsection{Real-time data analysis}

As soon as a buffer, containing the data of 500 events, is transferred to the PDP $11 / 60$ the computer starts the following analysis:

1) The time information is read and checked against a maximum value of $10 \mu \mathrm{s}$. 
2) The spatial coordinates of the STOP are next used to derive the $\mathrm{e}^{+}$track, which is checked to see if it intersects the sample volume.

3) The spatial coordinates of the last START are used to derive the $\mu^{+}$track which is checked to see it it intersects the sample volume.

4) The distance $d$ is evaluated and checked against a preset maximum value, and the coordinates of the vertex are compared with the sample position.

If the event fails eithex of the tests 1 and 2 , it is rejected. If it fails tests 3 or 4 the next to last START can be taken into consideration, if requested. The total time between the STOP and the new START is then evaluated and tests 1,3 , and 4 repeated. Tests 2 and 3 , which might seem to duplicate test 4 , are performed in advance of test 4 to avoid the time-consuming evaluation of the complete algorithm for patently spurious tracks.

It has been observed experimentally that about $10 \%$ of the accepted events were correlated with the second START, even though the average number of incoming muons was only $\sim 2 \times 10^{3}$ per second. This was due to the fact that the continuous beam partly retained the time structure of its original pulsed source. When an event passes all the tests it is stored in a temporary file in the central memory [9]. One or more data-acquisition routines can be activated at the same time: they have access to the temporary file according to a preselected priority and produce the time and spatial histograms. Finally, data may be analysed with programs to perform a fast Fourier transform, a norma1 Fourier transform, a least-squares fit and bidimensional and tridimensional plotting of the vertices. These programs work asynchronously with the data taking, which has the highest priority for the central processing unit (CPU).

\section{RESULTS}

A set of experiments was devised to determine the performance of the spectrometer. The first goal was to define the overall spatial and temporal resolution; the second was to test the spectrometer under the various conditions encountered in real experiments. 


\subsection{The spatial resolution and the temporal resolution}

The spatial resolution was determined experimentally. The muon beam was stopped in a graphite sample and the pseudo distances of all the collected events were evaluated. The distribution of these distances was plotted with a bin size of $0.5 \mathrm{~mm}$ up to a maximum value of $100 \mathrm{~mm}$. A Gaussian function was obtained with a half width at half height of $4 \mathrm{~mm}$ for one STOP telescope and of $5 \mathrm{~mm}$ for the other STOP telescope (one of these curves is plotted in fig. 5). Such a spread in the distribution of the pseudo distances is in agreement with the estimation obtained from simple geometrical considerations. Further estimates of both the track curvature in the low applied magnetic field (300 G) and multiple scattering effects within the sample lie well below this experimental value.

The time resolution was determined by means of $\mu$ SR experiments on a sample of fused quartz at room temperature. The amplitude of the muonium transitions in applied magnetic fields ranging from 6 to $120 \mathrm{G}$ was compared with the theoretical values. The results showed that the dominant contribution to the passband curve is the one from the TDC mentioned in Section 3.2.

\subsection{Recent applications}

The spectrometer has recently been used to study the transition to the antiferromagnetic ordering in some fluoride crystals.

The first material studied was $\mathrm{MnF}_{2}$. Previous experiments [10] in conventional spectrometers had shown that the free muon Larmor precession around the applied magnetic field, which is detected in the paramagnetic phase, drops sharply in amplitude from $11 \%$ to $3 \%$ in the ordered phase. It has already been pointed out in Section 2 that some fraction of this signal could be accounted for by muons stopping in the immediate neighbourhood of the sample, but it is important to verify whether muons from the $\mathrm{MnF}_{2}$ crystal itself contribute to the signal as well. A first $\mu \mathrm{SR}$ measurement was performed without constraints on the vertices; this showed the same precession as detected in the earlier experiments. It also provided the spatial reconstruction of the sample area, obtained by projecting the vertices onto the midplane, as seen in fig. 6a; this figure clearly shows the sample within the 
cryostat walls. Another measurement was made selecting only the decays from within the crystal: no Larmor precession was detectable in this case. The two histograms are shown in fig. $6 \mathrm{~b}$. It should be mentioned that a wire-chamber experiment provides histograms that begin exactly from the birth of the muon, while in a conventional experiment the anticoincidence veto used to eliminate spurious events can distort the data for as much as $200 \mathrm{~ns}$. Thus our apparatus is well suited to the study of signals with very fast damping (on the scale of the muon 1 ifetime), which are inaccessible to normal spectrometers. In the case under study it allowed us to rule out the possibility of a Larmor precession with a fast relaxation: in $\mathrm{MnF}_{2}$ no muons precess around the applied magnetic field below the Néel temperature and the $3 \%$ signal seen in earlier experiments came entirely from outside the sample.

Another experiment was performed, in which three different targets were mounted in the cryostat at the same time: a single crystal of $\mathrm{CoF}_{2}$, a single crystal of $\mathrm{SeF}_{2}$ and a $\mathrm{Cu}$ sample. While the latter provided a reference for amplitude and frequency calibration, it was possible to scan a wide range of temperatures of interest for the two fluorides: the wire-chamber spectrometer allowed separate reconstruction of the histograms collected from the different small samples, which would have stopped only a fraction of the beam if studied one by one.

A byproduct of these measurements was the calibration of the asymmetry parameter $\alpha[$ see Eq. (1)] for the present configuration. A value of $28 \%$ was obtained, which compares favourably with the $24 \%$ measured with scintillator counters only, in the same beam.

5. CONCLIJSIONS

The results described in the previous section are a definite confirmation that it is feasible to use wire chambers to reconstruct the $\mu^{+}-\mathrm{e}^{+}$vertex in "real time".

The read-out system can be improved. It can be considerably simplified by implementing hardware coding techniques directly on the wire-chamber circuit, as one of the authors has already show [4]. It can also be made much faster by 
substituting a microprocessor [11] for the PDP 11/04; this could also perform some of the preliminary tests on the coordinates that are executed at present by the $\operatorname{PDP} 11 / 60$.

Despite these limitations, which concern mainly the speed of data acquisition, the ability of the spectrometer to produce histograms without background is quite satisfactory. Moreover the possibility of discriminating between a sample and its surroundings gives a chance of carrying out an entirely new category of experiments that were extremely difficult to perform before. Examples of such experiments can be suggested by the measurements described in the last section, where several samples were irradiated by the muon beam at the same time. All experiments in which external conditions, such as temperature, need to be monitored with extreme precision to compare results of different samples are greatly facilitated.

We mention another type of experiment that has now become possible without a restrictive choice of the experimental conditions and sample compound as before [12]. That is high pressure experiments, where the pressure cell around the sample can stop enough muons to mask the signal from the real sample.

It is now evident that this type of spectrometer represents a significant advance in instrumentation for $\mu S R$ studies.

\section{Acknowledgements}

We should like to express our warm gratitude to the many people who helped us with valuable ideas and made the realization of this spectrometer possible. Among them we would particularly like to express our thanks to Mr. B. Lindberg who was the designer of the original read-out system, to Dr. G. Muratori who directed the construction of the wire chambers, to Mr. A. Domeniconi who looked after the chambers during and after their operational periods, and to Mr. C. Bizeau who implemented the data acquisition system and gave essential help to one of the authors. 
We are especially indebted to Professor C. Bucci who pushed us into this adventure and followed all the stages of the project, giving us much help and making invaluable suggestions. Finally we should like to thank the CERN Experimental Physics and Data Handling Divisions for general support. 


\section{REFERENCES}

[1] W.F. Lankford, K.G. Lynn, W.J. Kossler, A.T. Fiory, R.P. Minnich and C.E. Stronach, Nucl. Instrum. Methods 185 (1981) 469.

[2] F. Sauli, Report CERN 77-09 (1977).

[3] A. Jeavons, N. Ford, B. Lindberg, C. Parkman and Z. Hajduk, IEEE Trans. Nuc1. Sci. NS23 (1976) 259.

[4] A. Jeavons, K. Ku1l, B. Lindberg, G. Lee, D. Townsend, P. Frey and A. Donath, Nuc1. Instrum. Methods 176 (1980) 89.

[5] C. Engster and H. Verweij, Drift time digitizer DTD215, Provisional specification, Technical report CERN-EP/04-15 (1976).

[6] C. Engster and H. Verweij, Drift time digitizer DTD 164, Provisional adjustments and test procedures, Technical report, CERN-INP/06-20 (1975).

[7] S.F.J. Cox, A. Hill and R. De Renzi, J. Chem. Soc. Faraday Trans. 1, 78 (1982) 2975 .

[8] R. Tedeschi, Internal Report CERN-EP/80-03 (1980).

[9] R. Tedeschi, A new spectrometer for muon spin rotation, to appear in Proc. 1981 Enrico Fermi School of Physics, Varenna (Italy), 1981.

[10] R. De Renzi, R. Tedeschi, G. Guidi, C. Bucci and S.F.J. Cox, Solid State Commun. 43 (1982) 683.

[11] A. Jeavons, K. Hood, D. Townsend and P. Zwart, Proc. Topical Conf. on the Application of Microprocessors to High Energy Physics, Geneva, 1981 (CERN 81-07, Geneva, 1981), p. 276.

[12] T. Butz, J. Chappert, J.F. Dufresne, E. Karlsson, B. Lindgren, L.O. Norlin, P. Podini and A. Yaouanc, Phys. Lett. $\underline{75}$ (1980) 321. 


\section{Figure captions}

Fig. I : A typical uSR histogram: the amplitude of the modulation frequency here is $\sim 24 \%$ of the total, and the background is indicated by the dashed line.

Fig. 2 : a) Layout of one telescope (START) with wire chambers: DEG = degrader, $\mathrm{S}_{1} \mathrm{~S}_{2} \mathrm{~S}_{\mathrm{A}}=$ scintillator counters, $\mathrm{CO}=$ collimator, $\mathrm{W}_{1} \mathrm{~W}_{2}=$ wire chambers, (C) $D=$ (constant fraction) discriminators, $C A=$ charge amplifiers, $\mathrm{CP}=$ coordinate processors, $\mathrm{CC}=$ chamber controller, $\mathrm{FAN}=$ fan-in fan-out unit.

b) Schematic layout of a complete conventional spectrometer.

Fig. 3 : The block diagram of the data-collection system.

Fig. 4 : The pseudo distance $d \equiv|A-B|: m$ and $n$ are the muon and positron trajectories respectively.

Fig. 5 : The distribution $N(d)$ of the pseudo distances for events collected from a graphite sample in a $300 \mathrm{G}$ applied magnetic field.

Fig. 6 : a) Tridimensional (left) and bidimensional (right) plotting of the vertex distribution projected on the midplane. In both plots the cryostat walls are visible.

b) Muon spin rotation histograms from $\mathrm{MnF}_{2}$ at $200 \mathrm{G}$; the top one is obtained by relaxing the spatial constraints on the vertices. 


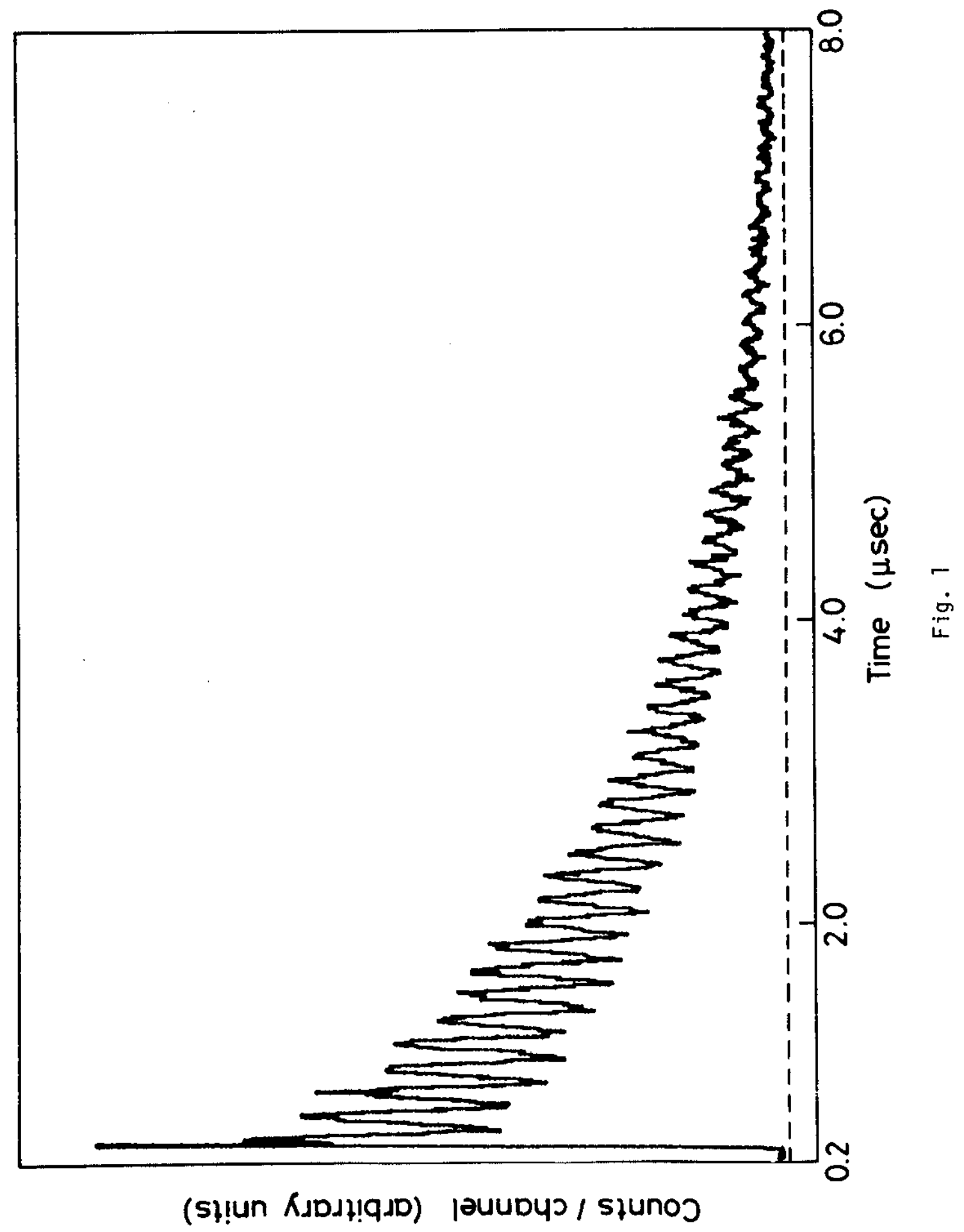




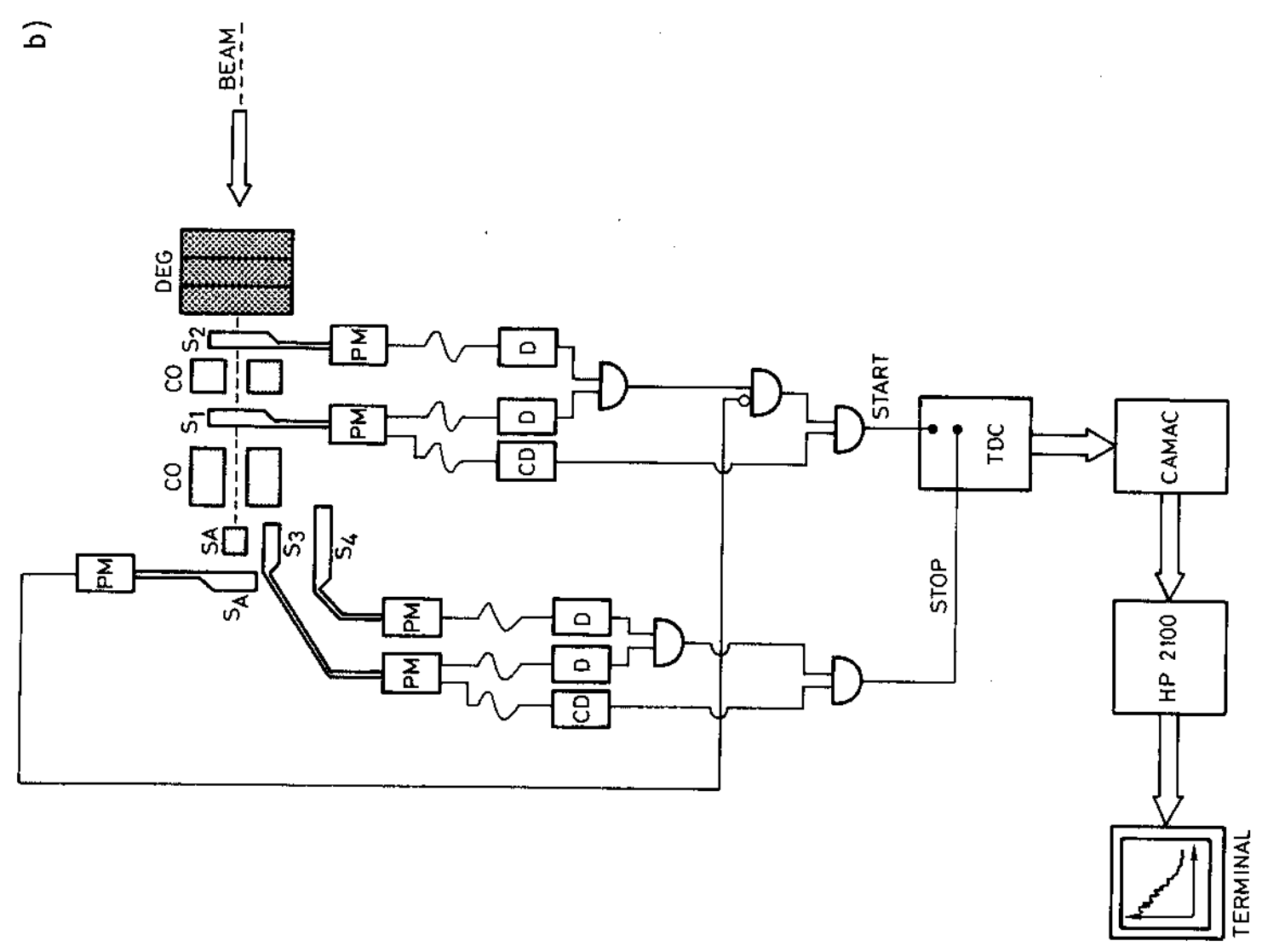

$\pi$

$\stackrel{\sim}{i}$

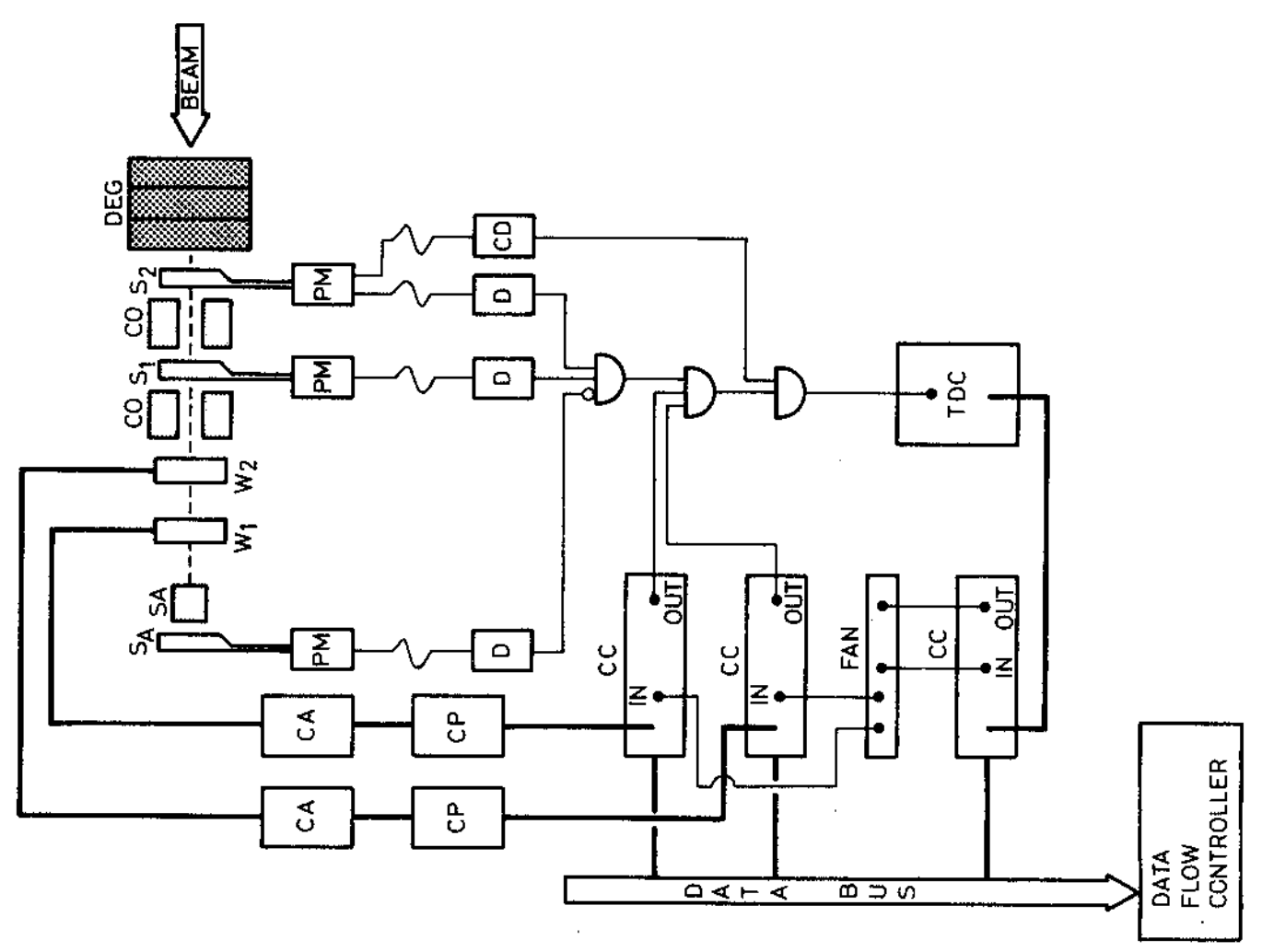




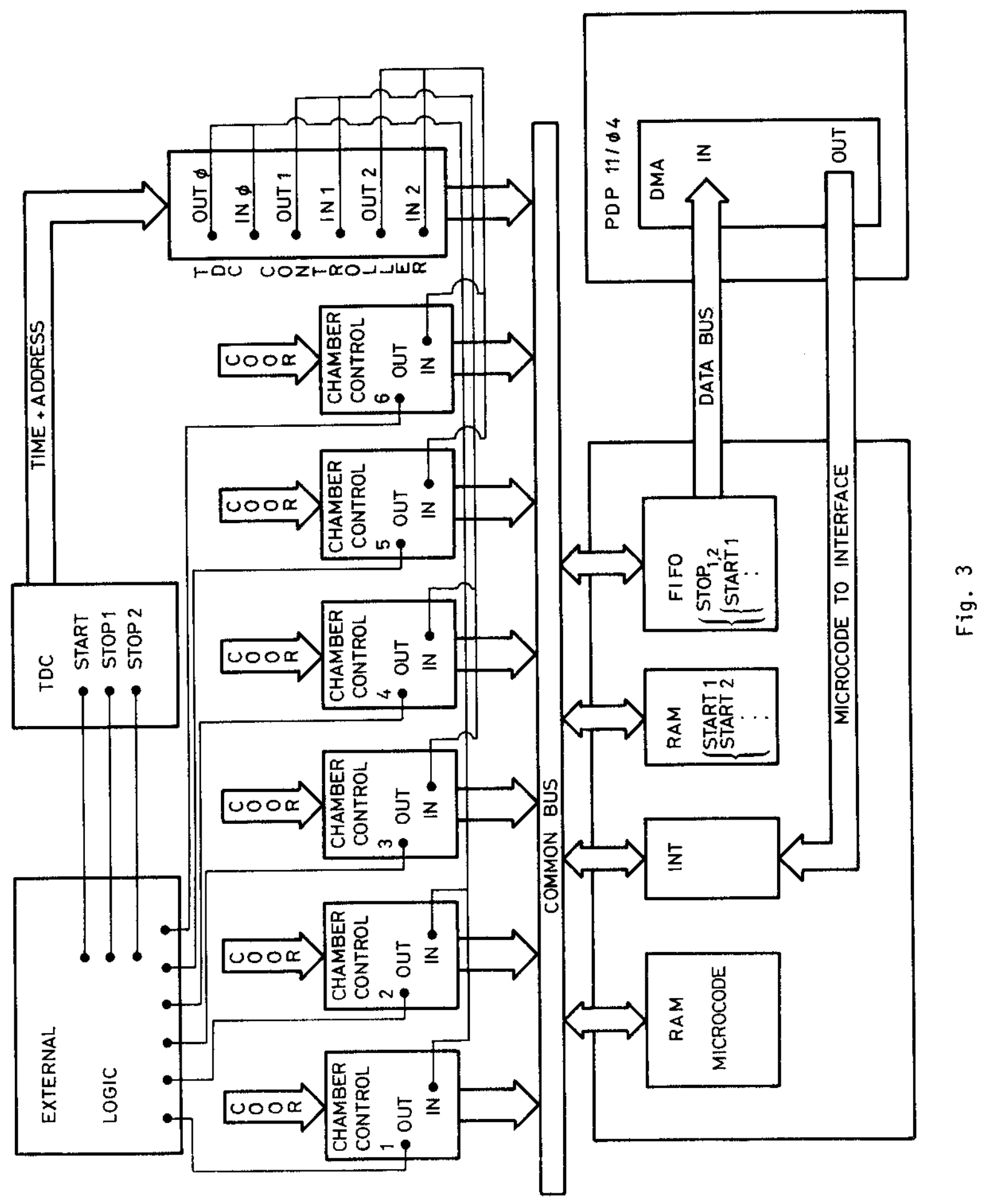




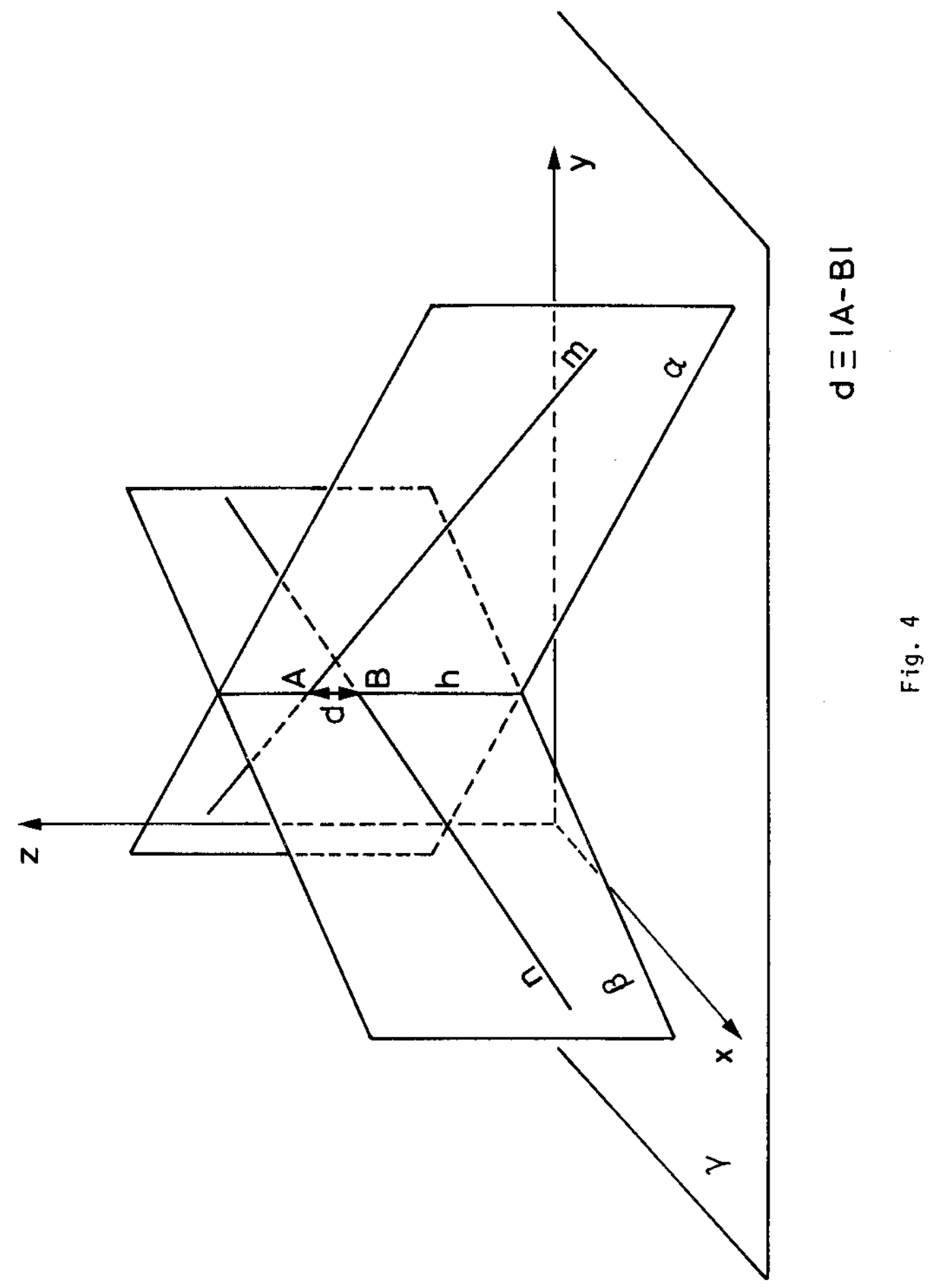




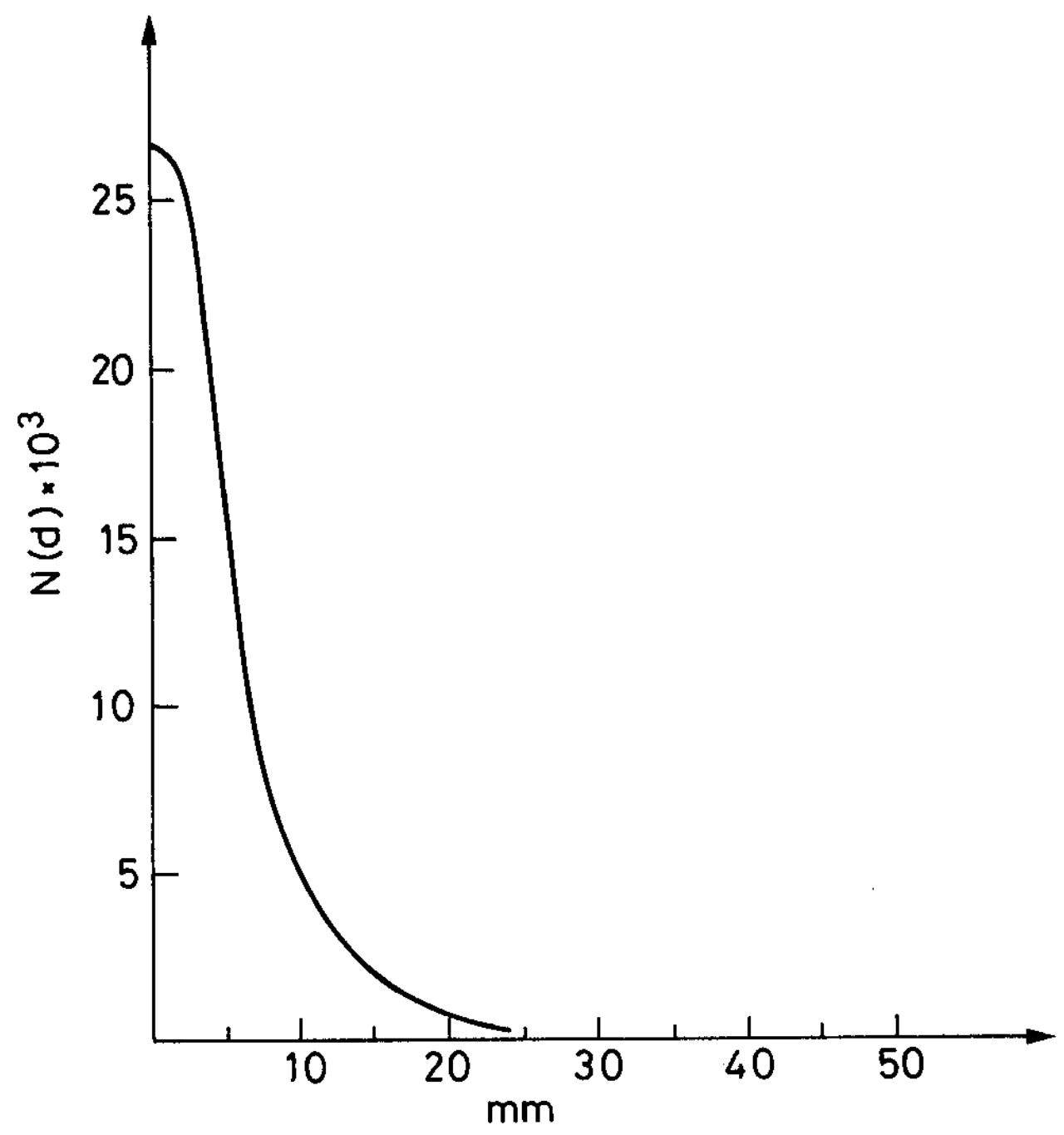

Fig. 5 
a)

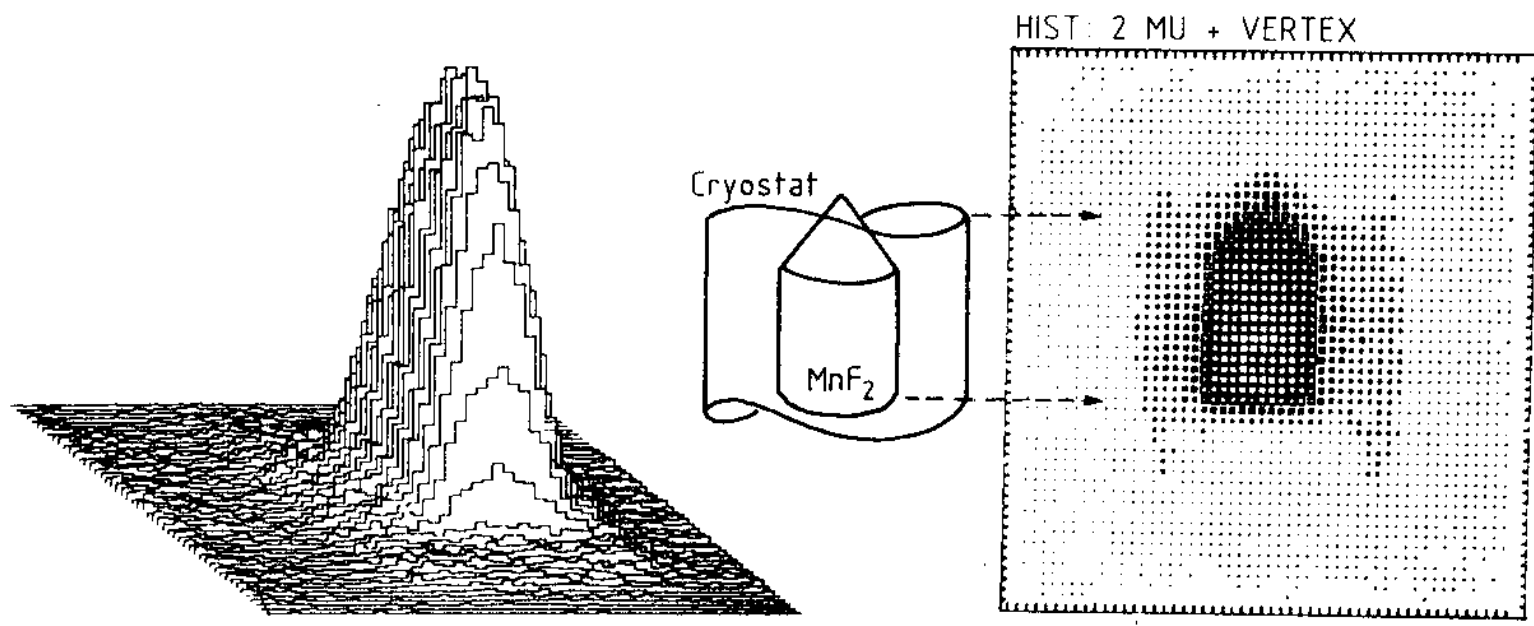

b)
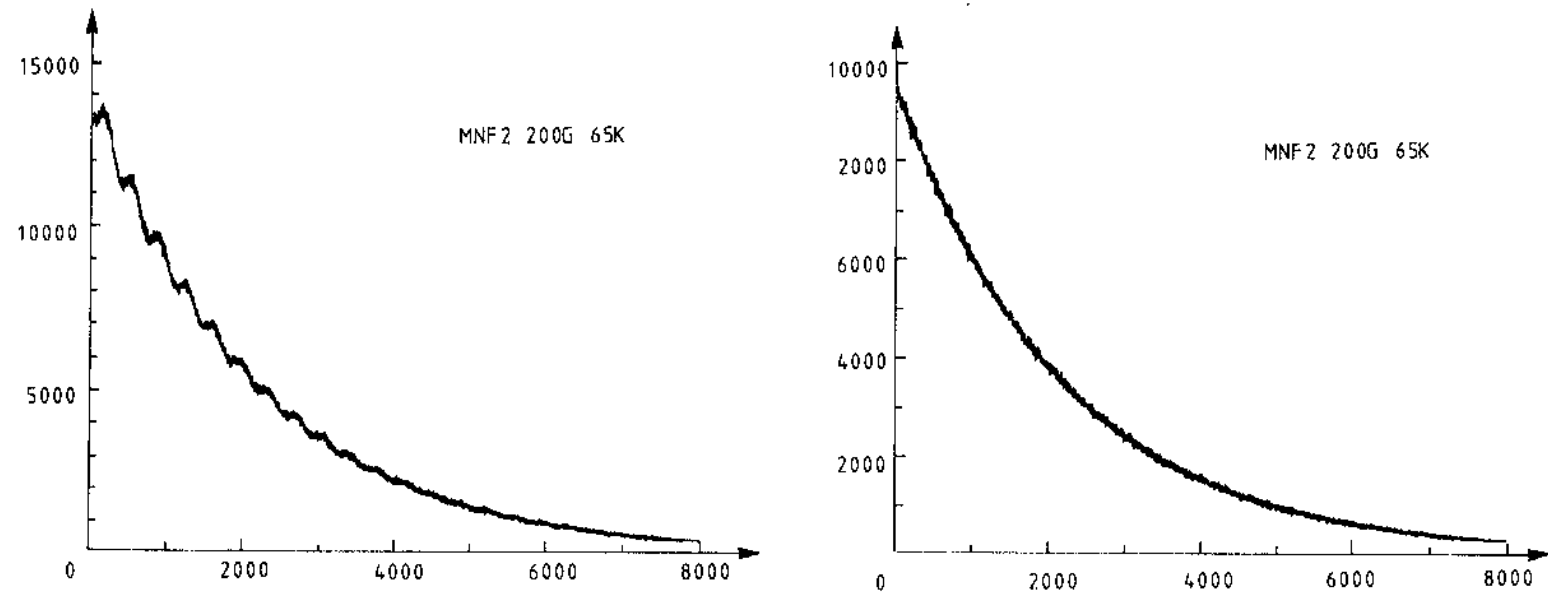

Fig. 6 
24 